\title{
Anti-inflammatory studies on a saponin albichinoside
}

\author{
Naresh Gupta ${ }^{1}$, Dinesh Kansal ${ }^{2 *}$, Anjna Sharma ${ }^{3}$ \\ ${ }^{1}$ Associate Professor, ${ }^{2}$ Professor and HOD, ${ }^{3}$ Tutor, Dept. of Pharmacology, ${ }^{1,3}$ Dr. Radhakrishnan Government Medical College Hamirpur, \\ Himachal Pradesh, ${ }^{2}$ Dr. Rajendra Prasad Government Medical College, Tanda, Himachal Pradesh, India
}

\section{*Corresponding Author: Dinesh Kansal}

Email: dinesh.kansal56@gmail.com

\begin{abstract}
Introduction: Pain and inflammation are a day to day affliction for all living beings. Mankind has for years been trying to relieve the agony of pain \& inflammation through various means with a large amount of success. Still we have not been able to produce the ideal analgesic or the ideal anti-inflammatory drug. The objective of this study was to evaluate a saponin, albichinoside, with respect to its ability to relieve pain and /or show anti-inflammatory activity.

Materials and Methods: The study was conducted on rats. The saponin was evaluated for its effects on acute, sub-acute, chronic and immunological inflammation by using standard anti-inflammatory study protocols.

Results: The test substance showed significant inhibition of acute, sub-acute and chronic inflammation produced by immunological and non-immunological methods in rats. These activities were qualitatively less as compared to indomethacin. This activity was like that of non-steroidal anti-inflammatory agents.

Conclusion: The test substance has moderate anti-inflammatory activity which could be by means of inhibition of prostaglandin synthesis and the complement.
\end{abstract}

Keywords: Saponin, Anti-inflammatory, Animals.

\section{Introduction}

Pain and inflammation are commonly encountered in our day to day life. Pain can be produced by a variety of causes and is an important part of inflammation. It affects our quality of life and capacity to perform. Since ancient times a variety of substances have been experimented with and used to ameliorate the agony of pain. In due course remedies were developed for treatment of various aspects of inflammation like swelling, rubor and warmth for which more potent drugs were developed. Age related deterioration and autoimmune diseases like osteoarthritis, spondylitis, rheumatoid arthritis etc. required stronger remedies, which have been developed from a variety of sources. But we have not been able to develop the ideal analgesic, anti-inflammatory or anti-arthritic so far. Towards this end an endeavor was made to look around us and tap a saponin for such a study. Albichinoside (ALB) a pure saponin fraction obtained from the bark and heartwood of a common plant Albizzia chinensis was taken up for this study.

\section{Plan of Study}

The effects of the test compound were evaluated for both non-immunological and immunological inflammation. The activity of albichinoside on acute inflammation was studied on carrageenin, histamine and serotonin induced hind paw oedema and on sub-acute inflammation by granuloma pouch and cotton pellet methods. For chronic inflammation formaldehyde induced arthritis method was used. Effects on immunological inflammation were evaluated by studies on adjuvant arthritis and acute tuberculin sensitivity reaction. The animals used for these studies were rats of the albino variety.

\section{Materials and Methods}

Albino rats which were used for the studies were housed in cages. The environment was kept clean, airy and warm. Sprouted green gram was fed to them routinely. Water was freely made available to them. The average weight of the rats was $120-225 \mathrm{gm}$. Animals of either sex but of the same strain were used. The drugs \& chemicals used for the studies were carrageenin, histamine, serotonin, croton oil, formaldehyde, Freund's adjuvant, PPD and betamethasone. The routes of administration were intraplantar, intradermal, intraperitoneal \& sub-cutaneous.

\section{Anti-inflammatory studies}

1. Non-immunological inflammation

a) Acute inflammation

i) Carrageenin-induced Hind Paw Oedema (Winter, Risley and Nuss, 1962). ${ }^{1}$ Rats were divided into three groups of five animals each. The hind paw volume of the animals was measured at the mid plantar region with a screw gauge. The drugs were then administered to the animals by the intra peritoneal route. The first group was given normal saline, the second indomethacin $(5.0 \mathrm{mg} / \mathrm{kg})$ and the third albichinoside $(5.0 \mathrm{mg} / \mathrm{kg})$, in a volume dose of $5.0 \mathrm{ml} / \mathrm{kg}$. One hour later carrageenin $1 \%$ was administered intra-plantarily in a common dose of $0.05 \mathrm{ml}$, to all the animals. For these injections a No. 26 H.D needle was used to minimize trauma.

ii) Histamine Induced Hind Paw Oedema (Parrat and West, 1958). ${ }^{2}$ The phlogistic agent used for this experiment was $0.1 \mathrm{ml}$ of $0.01 \%$ histamine. Otherwise, the experimental protocol followed was the same as for the carrageenin induced oedema. The dose of albichinoside was $5.0 \mathrm{mg} / \mathrm{kg}$ and of indomethacin $5.0 \mathrm{mg} / \mathrm{kg}$ too. The final paw volume was read at I hour. 
iii) Serotonin-induced Hind Paw Oedema (Parrat and West, 1958). ${ }^{2}$ The method was essentially the same as in case of carrageenin. The phlogistic agent used, however, was serotonin $(250 \mu \mathrm{g} / \mathrm{ml})$. The final paw volume was recorded after one hour. The doses of albichinoside and indomethacin used were $5.0 \mathrm{mg} / \mathrm{kg}$ each.

b) Sub-acute Inflammation

i) Granuloma Pouch Method (Selye, 1953). ${ }^{3}$ Healthy albino rats were injected subcutaneously with $5 \mathrm{ml}$ of air in between the scapula. Then $0.5 \mathrm{ml}$ of sterile $2 \%$ solution of croton oil in cotton-seed oil was injected into the air sac.

The animals were sacrificed on the $6^{\text {th }}$ day and the volume of the exudate in the sac was measured. The drug treatments with the vehicle, indomethacin and albichinoside were carried out from one day prior to the injection of phlogistic agent till the date of sacrifice.

ii) Cotton Pellet Method (Meier, Schuler and Desaulles, 1950). ${ }^{4}$ Sterilized cotton pellets weighing $10 \mathrm{mg}$ were implanted in both the axillae and groins under ether anesthesia in albino rats who had been divided into three groups. Pellets were removed on the $6^{\text {th }}$ day and kept overnight in an incubator at $37^{\circ} \mathrm{C}$. The pellets were weighed and weight of the granulation tissue was determined. The drug treatments with the vehicle, indomethacin and albichinoside were carried out from one day prior to the implantation of pellets till the day of sacrifice.

c) Chronic Inflammation

(i) Formaldehyde Induced Arthritis (Selye, 1949). ${ }^{5}$ Albino rats were divided into three groups. Formaldehyde induced arthritis was produced in the animals by injecting $0.1 \mathrm{ml}$ of $2 \%$ formaldehyde (v/v) into the hind paw under the plantar aponeurosis. The degree of arthritis induced was assessed by measuring daily the volume of the paw for 15 days. The percentage increase in paw volume was calculated for each day and the results were statistically analyzed.

\section{Immunological Inflammation}

i) Adjuvant Arthritis (Newbould, 1963). ${ }^{6}$

The animals (albino rats) were divided into three groups of 10 animals each. Adjuvant arthritis was induced in them by injecting $0.05 \mathrm{ml}$ of Freund's adjuvant containing a suspension of heat killed tubercle bacilli (human D.T.strain) in liquid paraffin $(5 \mathrm{mg} / \mathrm{ml})$. The degree of arthritis produced was assessed by measuring daily the volume of the paw for 13 days. The percentage increase in paw volume was calculated for each and the results statistically analyzed. The animals used in adjuvant arthritis were kept in metabolic cages and daily urine and faecal outputs measured. Subsequently they were killed on the $13^{\text {th }}$ day for further study. The severity of secondary lesions such as oedema of the injected hind paw and of the forepaws; dilatation of capillaries in the ears and nodules in the nail was estimated and categorized as mild, moderate and severe.

The animals were weighed daily and a record of their weight was maintained. Four groups of animals were used for this experiment. The various groups were injected with the vehicle, betamethasone and albichinoside respectively. ii) Acute Tuberculin Sensitivity Reaction (Satyavati, Prasad and Das, 1969b). ${ }^{7}$ This was performed on the $14^{\text {th }}$ day after injection of mycobacterium adjuvant. Purified tuberculin (P.P.D) was injected intradermally $(0.01 \mathrm{ml}$ of 1:10 dilution) into the flanks of the rats which were previously depilated. The diameter of the tuberculin reaction was measured 24 and 48 hours after injection. The drugs were administered 3 hours before injecting P.P.D.

\section{Observations and Results}

1) Carrageenin-induced Inflammation: Albichinoside produced statistically significant reduction in rat paw oedema $(p<0.001)$ in relation to control, though the effect was comparatively less than that of indomethacin $(\mathrm{P}<0.001)$. The percent inhibition of oedema with albichinoside was $60.8 \%$ which was less than that with indomethacin which caused $65.2 \%$ inhibition (Fig. 1).

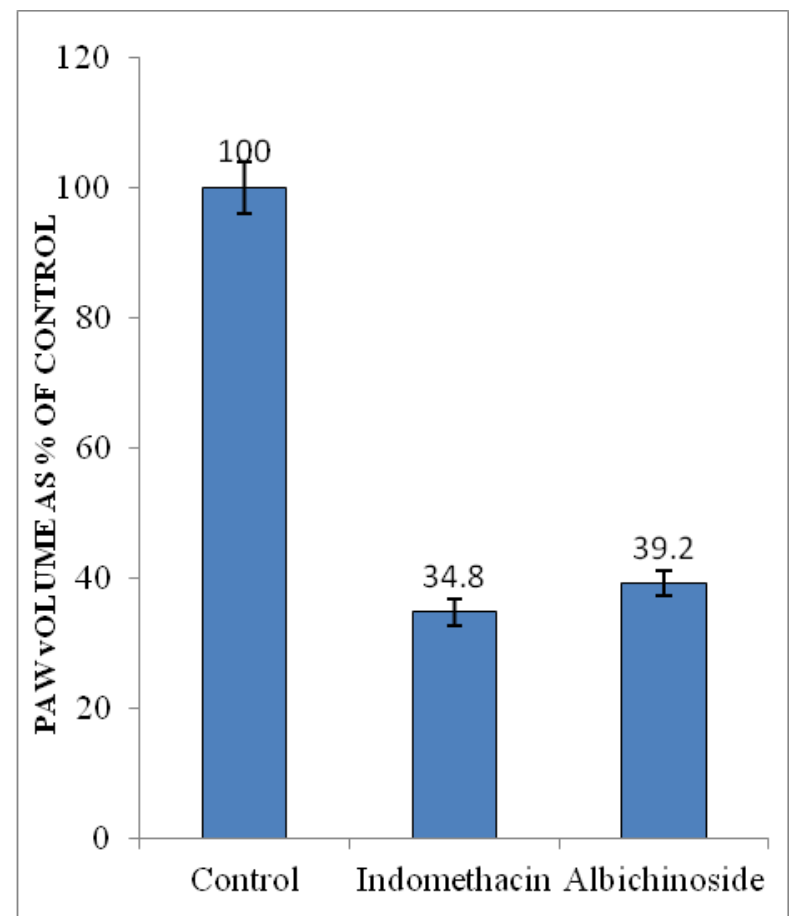

Fig. 1: Effect of albichinoside (ALB) and indomethacin on the carrageenin induced rat hind paw oedema

2) Histamine Induced Inflammation: Albichinoside produced a significant $(\mathrm{p}<0.01)$ reduction in rat hind paw oedema in this model. Indomethacin showed a potent effect too $(\mathrm{P}<0.001)$. The percent inhibition of oedema was $36.2 \%$ and $25.5 \%$ for indomethacin and albichinoside respectively (Fig. 2). 


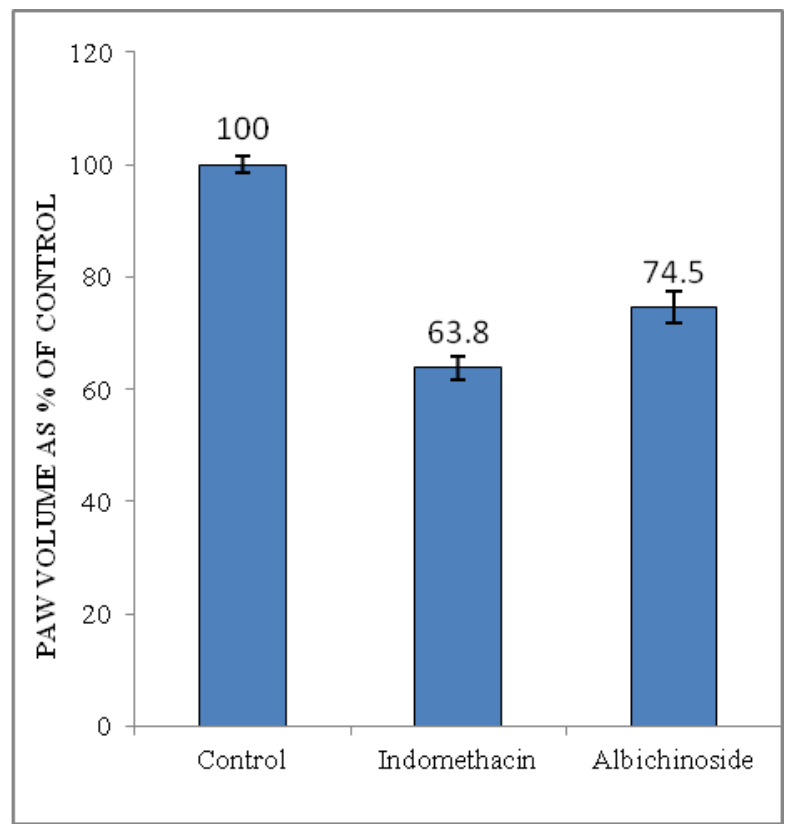

Fig. 2: Effect of Albichinoside (ALB) and indomethacin on histamine induced rat hind paw oedema

3) Serotonin Induced Oedema: Albichinoside produced a statistically significant reduction in rat paw oedema in this model which was comparable to that of indomethacin $(\mathrm{P}<$ 0.001 for both) in relation to control. Percent inhibition of oedema in case of albichinoside was $58.3 \%$ which was comparable to that of in indomethacin which showed $61.5 \%$ inhibition in relation to control (Fig. 3).

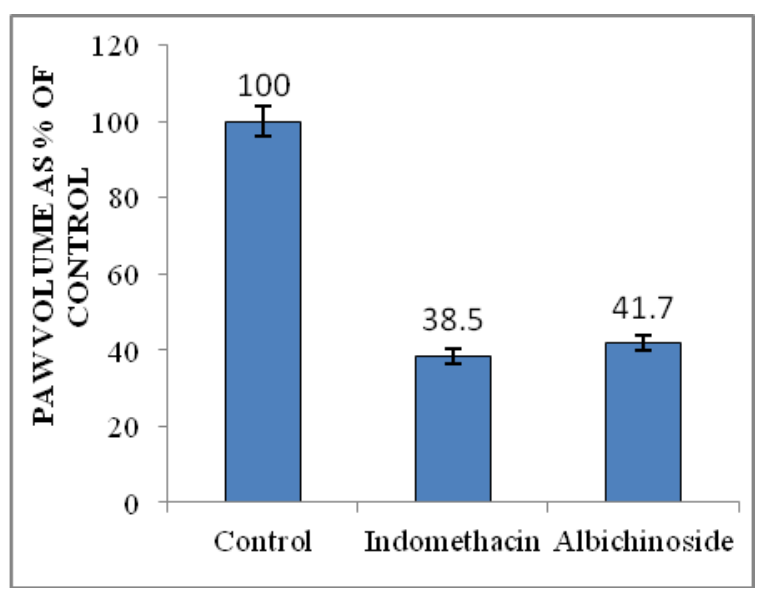

Fig. 3: Effect of albichinoside (ALB) and indomethacin on serotonin induced rat hind paw oedema

4) Granuloma Pouch Method: Albichinoside produced significant reduction in the volume of the exudate to the extent of $20 \%$. Indomethacin was slightly more effective, producing a $30 \%$ reduction in exudate volume $(\mathrm{p}<0.02)$. Mean volume of inflammatory exudate $(\mathrm{ml} / 100 \mathrm{gm}$ body wt.) was $0.50 \pm 0.025$ (Mean \pm SE), $0.35 \pm 0.025$ and $0.40 \pm$ 0.025 in the control, indomethacin treated and albichinoside treated groups (Fig. 4).

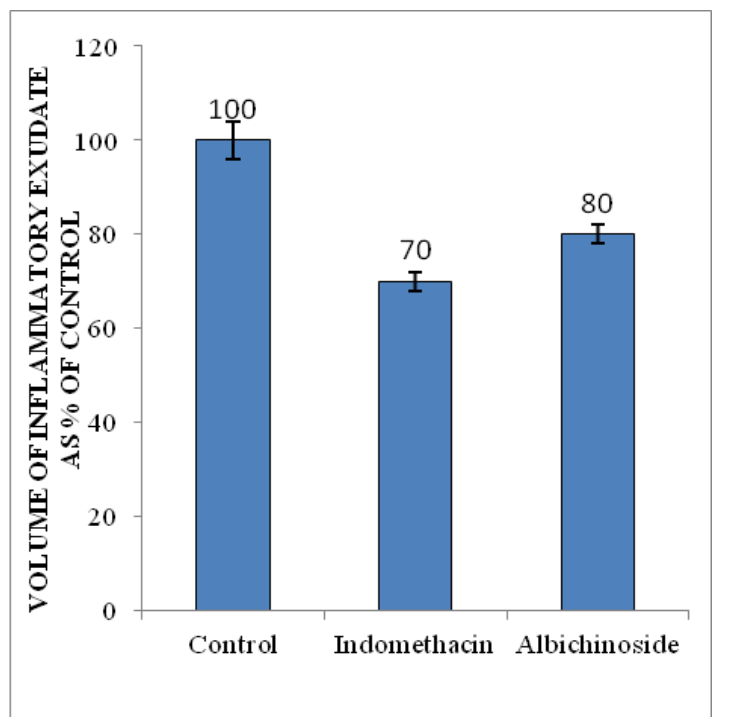

Fig. 4: Effect of albichinoside (ALB) and indomethacin on the inflammatory exudate in the granuloma pouch in the rat

5) Cotton pellet Granuloma: Albichinoside, as well as, indomethacin significantly reduced the formation of granuloma tissue. The mean weight of granulation tissue (mg/100 gm body weight) in the control group was $80 \pm 8.0$ in the indomethacin-treated group $40 \pm 5.5$ and in the albichinoside treated group 50 \pm 5.0 Indomethacin $(\mathrm{P}<0.001)$ was slightly more potent as compared to albichinoside $(\mathrm{P}<0.005)$ (Fig. 5).

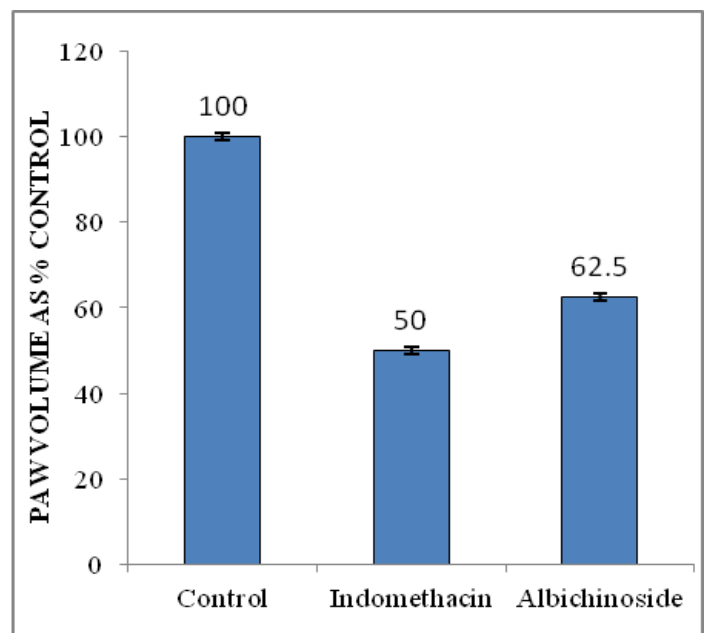

Fig. 5: Effect of albichinoside (ALB) and indomethacin on the granulation tissue formation in the cotton pellet granuloma in the rat

6) Tuberculin Sensitivity Reaction: Albichinoside significantly inhibited the tuberculin sensitivity reaction at 24 hours $(\mathrm{P}<0.05)$ and at 48 hours $(\mathrm{P}<0.002)$ while indomethacin did so only at 24 hours $(\mathrm{P}<0.005)$. The mean diameter of erythema $(\mathrm{mm})$ in control, indomethacin treated and albichinoside treated groups was $14.0 \pm 2.0,4.5 \pm 1.0$ and $9.8 \pm 1.4$ at 24 hours and $12.0 \pm 2.0,8.0 \pm 1.0$ and $5.0 \pm 1.0$ at 48 hours respectively (Fig. 6). 


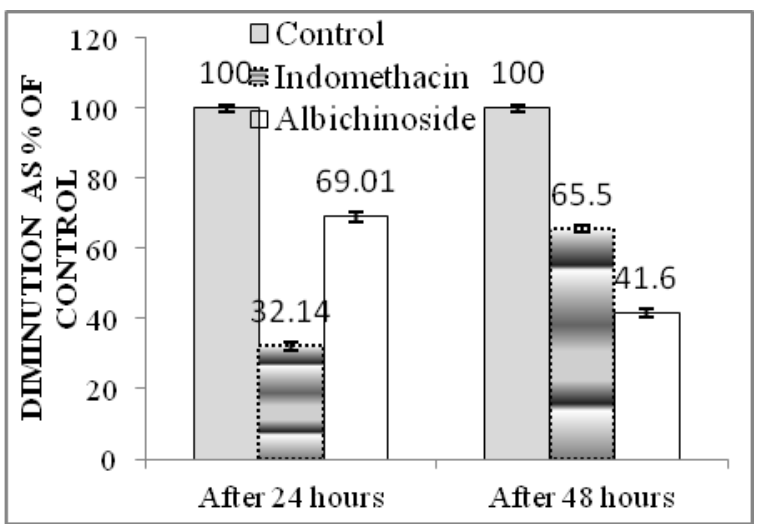

Fig. 6: Effect of albichinoside (ALB) and indomethacin on the acute tuberculin sensitivity reaction in albino rats

7) Formaldehyde Induced Arthritis: Albichinoside $(\mathrm{P}<0.001)$ and indomethacin $(\mathrm{P}<0.001)$ both significantly decreased the paw volume. The percent increase in foot volume in the control group reached the maximum on the $11^{\text {th }}$ day $(160 \pm 14.5)$ and subsequently there was a decrease on the $13^{\text {th }}$ day $(150 \pm 3.5)$ and $15^{\text {th }}$ day $(150 \pm 10.5)$. In the indomethacin treated group the maximum increase in paw volume was seen on the $5^{\text {th }}$ day $(30 \pm 6.5)$ which declined by the $9^{\text {th }}$ day $(10 \pm 5.5)$ and continued at this level up to the $15^{\text {th }}$ day. The animals treated with albichinoside showed the maximum paw volume on the 7 th day $(60 \pm 1.0)$ which declined by the $15^{\text {th }}$ day $(50 \pm 2.5)$ (Fig. 7).

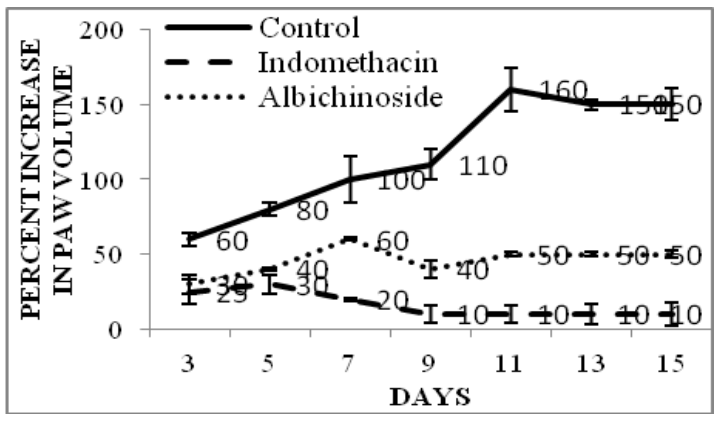

Fig. 7: Effect of albichinoside (ALB) and indomethacin on formaldehyde induced arthritis in albino rats

8) Adjuvant Arthritis: Maximum swelling in the control group was seen on $5^{\text {th }}$ day $(75 \pm 12.0)$ which declined slightly on the $7^{\text {th }}$ day $(70 \pm 10.0)$ but it again increased from $9^{\text {th }}$ day $(90 \pm 12.5)$ onwards reaching its maximum on the $13^{\text {th }}$ day $(110 \pm 13.0)$. Indomethacin significantly suppressed only the primary phase of swelling ( $1^{\text {st }}$ to $5^{\text {th }}$ day). Whereas both albichinoside and betamethasone significantly suppressed both the primary and secondary phases of the swelling. The maximum paw volume was on the $1^{\text {st }}$ day $(30 \pm 5.5)$ and it declined gradually to $(10 \pm 3.0)$ by the $13^{\text {th }}$ day.

In addition, in control group a swelling was also noticed in the uninfected paw, as well as, in the fore-paws on the $9^{\text {th }}$ day. Furthermore, inflammatory lesions were also observed in the tail in the form of nodules which often ulcerated, and in the ears as dilated capillaries. These secondary lesions were graded as mild, moderate and severe. The nature of secondary lesion was modified from severe degree to moderate or mild by albichinoside and betamethasone whereas indomethacin did not have any effect (Fig. 8).

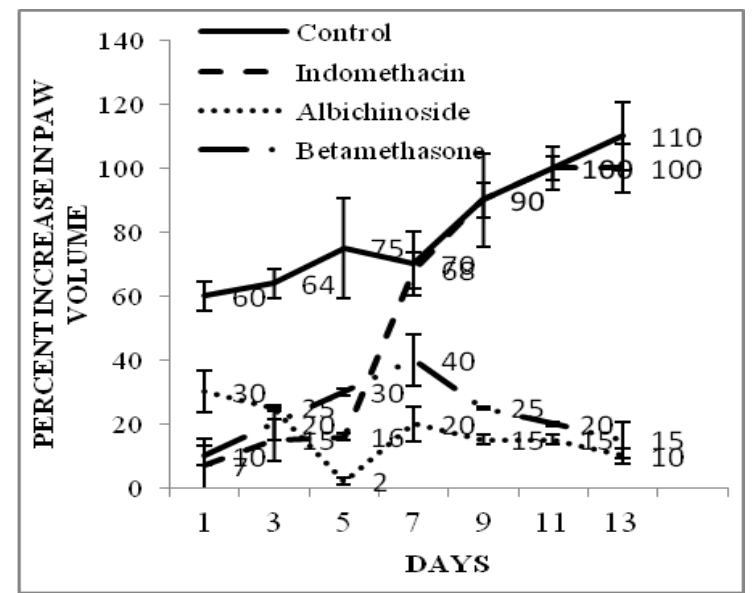

Fig. 8: Effect of albichinoside (ALB), betamethasone and indomethacin on adjuvant arthritis in albino rats

\section{Conclusion}

The compound significantly inhibited acute, sub-acute and chronic inflammation produced by both immunological and non-immunological methods in albino rats. But the antiinflammatory and anti-arthritic activities of albichinoside were found to be less than those of indomethacin.

The available evidence suggests that the antiinflammatory and anti-arthritic activities were like those of the non-steroidal anti-inflammatory agents. The possibility of albichinoside causing inhibition of prostaglandin synthesis and the complement cannot be ruled out.

\section{References}

1. Winter, C.A., Risley. E.A. and Nuss, G.W. Proc. Soc Exp Biol. (N.Y.), 1962;111:544.

2. Parratt, J.R. and West, G.B. (1958). Brit J Pharmacol. 1958;13;65.

3. Selye, H. J Amer Med Ass. 1953;152:1207.

4. Meier, R., Schuler, W. and Desaulles, P. Experientia (Basel), 1950;6:469.

5. Selye, H. Brit Med J., 1949;4:1129.

6. Newbould, B.B (1963). Brit J Pharmacol. 1963;1:127.

7. Satyavati, G.V., Prasad, D.N. and Das, P.K. Indian J Physiol Pharmacol. 1969;13:37.

How to cite this article: Gupta N, Kansal D, Sharma A. Anti-inflammatory studies on a saponin albichinoside. Indian J Pharm Pharmacol. 2018;5(4):191-194. 